\title{
Should anaesthesia method for prostate biopsy be the same for every patient? A randomised prospective study to determine the risk factors for pain
}

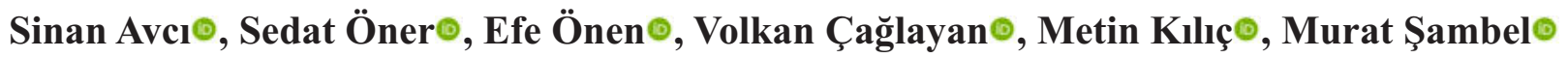 \\ Department of Urology, University of Health Sciences, Bursa Yüksek İhtisas Training and Research Hospital, Bursa, Turkey
}

\begin{abstract}
Objectives: To evaluate the risk factors for pain occurring during prostate biopsy.

Methods: This study included 123 patients were applied with prostate needle biopsy under transrectal ultrasonography. The patients were randomly separated into 3 groups of 41 individuals. For periprostatic nerve blockage, $10 \mathrm{cc} 2 \%$ lidocaine was applied to Group 1, $10 \mathrm{cc} 0.25 \%$ levobupivacaine to Group 2, and $10 \mathrm{cc}$ $0.25 \%$ bupivacaine to Group 3. A $10 \mathrm{~cm}$ Visual Analogue Scale (VAS) was used to evaluate patient pain. The pain of the patients was evaluated in 4 stages. VAS 1: Pain score during the injection of the anaesthetic agent; VAS 2: Pain score during the biopsy when half the procedure was completed; VAS 3: Pain score following removal of the rectal probe immediately after the biopsy; and VAS 4: Pain score at 1 hour after the biopsy.

Results: There were significant negative correlations between VAS 3 pain scores and age in group 1, group 3 and for entire cohort ( $p=0.013, p=0.031$ and $p=0.033$, respectively). In group 1 both total and free PSA showed significant negative correlations with VAS 3 pain scores $(p=0.020$ and $p=0.010$, respectively). In group 2 VAS 4 pain scores of the patients with suspicious digital examination findings were found to be significantly higher than those of the patients with benign digital examination findings $(p=0.025)$.

Conclusions: Of all patients to be applied with prostate biopsy, those of a younger age, with a lower PSA level, with suspicious digital rectal examination findings constitute a relatively higher risk group in respect of pain.

Keywords: biopsy, pain, prostate, risk factors
\end{abstract}

$\mathrm{T}$ he current standard method used to determine prostate cancer is prostate biopsy applied under transrectal ultrasonography (TRUS) guidance. When automatic biopsy instruments started to be used in prostate biopsy under TRUS guidance, patient comfort increased as the procedure became quicker and the needles are finer [1]. However, despite these developments, several studies have reported that the majority of patients feel discomfort because of pain felt during the biopsy $[2,3]$. Many different protocols have been used in an attempt to control pain, ranging from minimally invasive methods such as the use of non-steroid anti-inflammatory drugs or rectal administration of an anaesthetic agent, to relatively more invasive methods such as periprostatic nerve blockage, or pudendal block. At this point, it is important to identify which anaesthesia methods will be more effective on which patient groups, or in the selection of how invasive an anaesthesia method will be in a specific patient group, the risk factors that could cause pain. The aim of the 
current study was to evaluate the risk factors for pain occurring during prostate biopsy.

\section{METHODS}

Approval for the study was granted by the Local Ethics Committee (decision no: 2012/9/3) and signed informed consent was obtained from all the patients. This study included 123 patients were applied with prostate needle biopsy under transrectal ultrasonography (TRUS) guidance because of suspected prostate cancer. The patients included in the study comprised those with indications for prostate biopsy of abnormal rectal examination findings and/or serum PSA levels $>2.5 \mathrm{ng} / \mathrm{mL}$. Patient age, total and free PSA levels, prostate volume, education level, digital rectal examination findings, pathology and biopsy-related complications results were recorded.

In respect of the level of education of the patients, they were separated as 8 years of compulsory education or less (primary school and below) and more than 8 years of compulsory education (above primary school). The digital rectal examination findings of the patients were evaluated as benign or suspicious. Patients with findings of hardness, nodule, irregularity or eradication of the sulcus in the digital rectal examination were classified as suspicious. The pathology results of the patients were recorded as benign or malignant.

By adding new patients to the subsequent group, the patients were randomly separated into 3 groups of 41. For periprostatic nerve blockage, 10 cc $2 \%$ lidocaine was applied to Group 1, 10 cc $0.25 \%$ levobupivacaine to Group 2, and $10 \mathrm{cc} 0.25 \%$ bupivacaine to Group 3.

The patients were positioned in the left lateral decubitus position with the hips and knees in flexion. For the TRUS imaging, a ultrasound device was used with a $6.5 \mathrm{mHz}$ rectal probe of the widest diameter of $23 \mathrm{~mm}$ (LOGIQ 100 PRO Series). Following rectal placement of the probe, the prostate was visualised in the sagittal and transverse planes and prostate volume was automatically calculated with the ellipsoid formula in the ultrasound machine.

Following aspiration to prevent intravascular injection, the anaesthetic agents were injected slowly using a $30 \mathrm{~cm} 18$ gauge $(\mathrm{G})$ spinal needle, as two separate $5 \mathrm{cc}$ doses between the prostate floor and the seminal vesicle in the sagittal plane to the area where both neurovascular bundles are. When the periprostatic nerve blockage was obtained, biopsy samples were taken from each patient as a standard 12-core biopsy from the posterolateral region of the peripheral zone, in accordance with the European Association of Urology (EAU) guidelines, using a 30 $\mathrm{cm} 18 \mathrm{G}$ fully automatic biopsy needle. As this was the first biopsy for all the patients in this study, transitional zone sampling was not applied. In all the patients, all the 12-core biopsy samples were taken following the same anatomic sequence.

Starting 1 day before the biopsy procedure and continuing for 4 days after, all patients were administered oral $500 \mathrm{mg}$ ciprofloxacin twice a day. To clean the intestines, Fleet enema was administered intrarectally on the morning of the biopsy.

A $10 \mathrm{~cm}$ Visual Analogue Scale (VAS) was used to evaluate patient pain. The scale was explained to the patients and they were instructed to mark the scale to represent their pain where $0=$ no pain and $10=$ the most severe pain ever experienced. Data obtained by measuring in millimeters the marks made on the scale by the patient were recorded as the pain scores.

The pain of the patients was evaluated in 4 stages. VAS 1: Pain score during the injection of the anaesthetic agent; VAS 2: Pain score during the biopsy when half the procedure was completed; VAS 3: Pain score following removal of the rectal probe immediately after the biopsy; and VAS 4: Pain score at 1 hour after the biopsy. Explaining VAS to patients, recording VAS scores and digital rectal examination findings, and all biopsies were performed by the same physician (SA).

Patients were monitored for 1 hour after the procedure and any complications were recorded. Those with no complications were discharged. Second evaluations related to complications were made during the follow-up visits for the pathology results. Complications without any medical or surgical interventions were evaluated as minor complications. The opposite was evaluated as major complications. Complications were also evaluated according to Clavien-Dindo classification.

\section{Statistical Analysis}

As the variables did not conform to normal 
distribution, comparisons were made with nonparametric statistical tests. In the comparisons between groups, the Mann-Whitney and KruskalWallis tests were used, and for categorical variables, the Chi-square and Fisher tests. Correlations between VAS values and quantitative data were evaluated with Spearman analysis. As non-parametric tests were used, the results were stated as median, minimum and maximum values. A value of $p<0.05$ was accepted as statistically significant.

\section{RESULTS}

The median, minimum and maximum values of patient age, total PSA, free PSA and prostate volume of the groups are shown in Table 1. No statistically significant difference was determined between the groups in respect of age, total PSA, free PSA and prostate volume $(p>0.05)$ (see Table 1). The education level, digital rectal examination findings and pathology results of the groups are shown in Table 1. No statistically significant difference was determined between the groups in respect of education level, digital rectal examination findings and pathology results $(p>0.05)$. The median, minimum and maximum values of the VAS scores calculated according to the education level, digital rectal examination findings and pathology results are shown in Table 2.

In Group 1 and Group 3, and for entire cohort, a statistically significant negative correlation was determined between age and the VAS 3 pain scores (correlation coefficients: $-0.388,-0.337,-0.192$, respectively, $p<0.05$ ) (Table 3). In Group 2, no statistically significant correlation was determined between age and any of the VAS scores $(p>0.05)$. A statistically significant negative correlation was determined between total PSA and the VAS 3 score in Group 1 (correlation coefficient: $-0.367, p=0.020$ ). In Group 2, Group 3, and for entire cohort, no statistically significant negative correlation was determined between total PSA and pain scores $(p<$ 0.05) (see table 3).

In Group 1, a statistically significant negative correlation was determined between free PSA and

Table 1. The comparisons between the groups of the age, total PSA, free (PSA) and prostate volume of the patients

\begin{tabular}{|c|c|c|c|c|}
\hline & Group 1 & Group 2 & Group 3 & $p$ value \\
\hline Age, years & $66.5(50-79)$ & $62(50-83)$ & $64(49-82)$ & 0.057 \\
\hline Total PSA & $7.3(0.9-100)$ & $7(1.4-49)$ & $11.2(3.4-314)$ & 0.202 \\
\hline Free PSA & $1.5(0.2-50)$ & $1.3(0.3-6.4)$ & $1.6(0.3-66)$ & 0.127 \\
\hline Prostate volume & $65.5(21-160)$ & $57(20-122)$ & $63(13-240)$ & 0.233 \\
\hline \multicolumn{5}{|l|}{ Education level } \\
\hline $\begin{array}{l}\text { Primary school and below } \\
(\mathrm{n}=95)\end{array}$ & $30(73 \%)$ & $32(78 \%)$ & $33(80 \%)$ & 0.376 \\
\hline $\begin{array}{l}\text { Primary school and above } \\
(\mathrm{n}=28)\end{array}$ & $11(27 \%)$ & $9(22 \%)$ & $8(20 \%)$ & \\
\hline \multicolumn{5}{|l|}{ Digital rectal examination } \\
\hline Benign $(n=69)$ & $25(61 \%)$ & $25(61 \%)$ & $19(46 \%)$ & 0.147 \\
\hline Suspicious $(n=54)$ & $16(39 \%)$ & $16(39 \%)$ & $22(54 \%)$ & \\
\hline \multicolumn{5}{|l|}{ Pathology result } \\
\hline Benign $(\mathrm{n}=94)$ & $32(78 \%)$ & $30(73 \%)$ & $32(78 \%)$ & 0.862 \\
\hline Malignant $(n=24)$ & $9(22 \%)$ & $11(27 \%)$ & $9(22 \%)$ & \\
\hline
\end{tabular}

Data are shown as median (minimum-maximum) or $\mathrm{n}(\%)$. The $p$ values was calculated with the Kruskal Wallis test. The $p$ values of the comparisons with the Chi-square test betwen the groups and of the numerical distribution of the education level, digital rectal examination findings and pathology results. PSA $=$ prostate specific antigen 


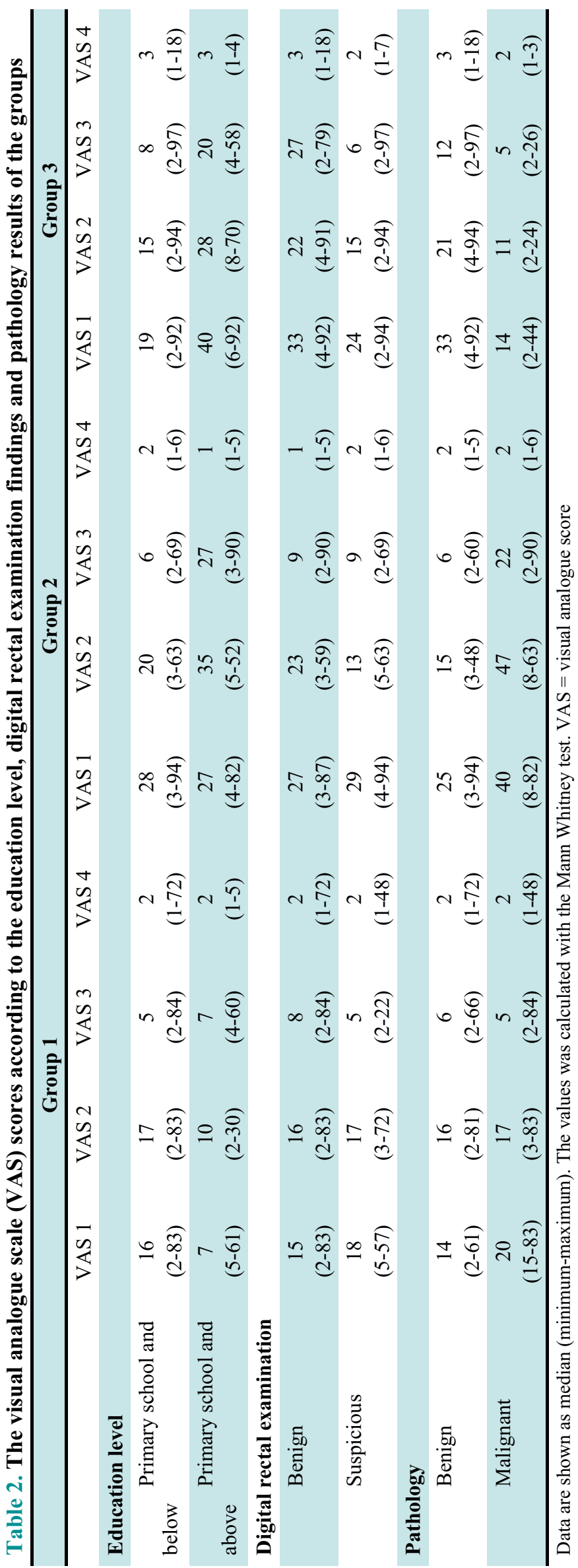

VAS 3 pain scores. For entire cohort, a statistically significant negative correlation was determined between free PSA and VAS 2 pain scores (correlation coefficients: $-0.401,-0.185$, respectively). In Group 2 and Group 3, no statistically significant finding was recorded between free PSA and any of the pain scores. No statistically significant result was obtained in any of the groups between prostate volume and any of the pain scores. The $p$ values calculated for the correlations between pain scores and age, total PSA, free PSA and prostate volume values of the groups separately and together are shown in Table 3.

When the groups were evaluated separately and together, education level was not determined to have significantly affected the pain scores. No statistically significant effect on the pain scores was seen of the digital rectal examination findings in Group 1, Group 3 and for entire cohort. In Group 2, the digital rectal examination findings were observed to have a significant effect on the VAS 4 score. The pain scores of the group with suspicious examination findings were found to be significantly higher than those of the group with benign examination findings.

In Group 1 and for entire cohort, the pathology results were not observed to have had a significant effect on the pain scores. In Group 2, the pathology results were determined to have had a significant effect on the VAS 2 pain scores, and in Group 3 on all the pain scores. In Group 2, the pain scores of those with malignant pathology results were significantly higher than those of the patients with benign results. In Group 3, the pain scores of those with benign pathology results were significantly higher than those of the patients with malignant pathology results. The $p$ values calculated for the effects on pain scores of education level, digital rectal examination findings and pathology results of the groups separately and together are shown in Table 3.

As minor complications, rectal bleeding was seen in 35 patients and hematuria in 10 patients. The only major complication was orchitis observed in 1 patient. Rectal hemorrhage and hematuria resolved spontaneously without any surgical or medical intervention so they were classified as grade 1 according to Clavien Dindo classification. Because of medical treatment orchitis was classified as grade 2 according to Clavien Dindo classification. The distribution of complications according to the groups 


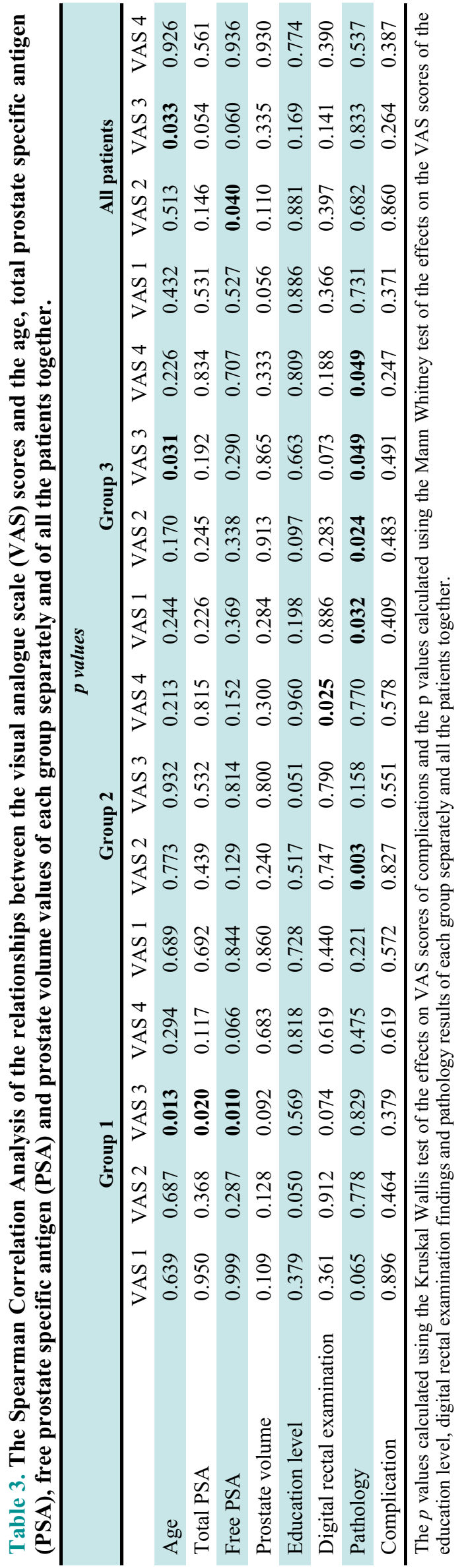

is shown in Table 4. In the paired comparisons of the groups, no significant difference was determined in respect complications ( $\mathrm{p}$ values are shown in Table 4). When the groups were evaluated separately and together, no significant correlation was seen between pain and complications ( $\mathrm{p}$ values are shown in Table $3)$.

When the pain score results of all the groups were evaluated together according to the education level, digital rectal examination findings and pathology results and the pain score results in each evaluation according to complications were not statistically significant, they are not shown in Table 2 . The $p$ values related to the above-mentioned evaluations are shown in Table 3.

There was no statistically significant difference between the groups for VAS 1, VAS 2 and VAS 3 pain scores. Since there was a statistically significant difference between the groups for VAS 4 pain scores, pairwise comparisons were examined between the groups ( $\mathrm{p}$ values are shown in Table 5 and Table 6).

\section{DISCUSSION}

Prostate biopsy applied under transrectal ultrasonography (TRUS) guidance remains the current standard method used in the diagnosis of prostate cancer. Many studies have been conducted to reduce the pain that occurs associated with this procedure and the necessity for the application of anaesthesia before prostate biopsy has been included in the guidelines. However, few studies have evaluated the risk factors for pain. In the European Association of Urology (EAU) guidelines there is no mention of in which patient groups pain may develop in particular and the same anaesthesia method is recommended for all patients.

In the current study, a statistically significant negative correlation was found between age and the pain scores measured immediately after the procedure (VAS 3) in Group 1, Group 3 and for entire cohort. According to this, the pain scores were significantly higher in younger patients. This negative correlation showed a similarity with several studies in literature [1, 4-7]. Djavan et al. [4] stated that significantly greater pain was felt by patients aged $<60$ years compared to older patients. This can be considered to 
Table 4. Distribution of complications in the groups

\begin{tabular}{|c|c|c|c|c|c|c|c|c|c|}
\hline & \multicolumn{6}{|c|}{$\begin{array}{l}\text { Minor complication } \\
\text { (Clavien Dindo group 1) }\end{array}$} & \multirow{2}{*}{\multicolumn{3}{|c|}{$\begin{array}{c}\begin{array}{c}\text { Major complication } \\
\text { (Clavien Dindo group 2) }\end{array} \\
\text { Orchitis }\end{array}$}} \\
\hline & \multicolumn{3}{|c|}{ Rectal bleeding } & \multicolumn{3}{|c|}{ Hematuria } & & & \\
\hline & $\begin{array}{l}\overrightarrow{0} \\
\text { D } \\
\text { 安 }\end{array}$ & $\begin{array}{l}\overrightarrow{0} \\
\text { D. } \\
\text { D. } \\
\end{array}$ & $\begin{array}{l}\stackrel{0}{\Xi} \\
\stackrel{\pi}{2} \\
\Omega\end{array}$ & $\begin{array}{l}\overrightarrow{0} \\
0 \\
\text { D } \\
\dot{z}\end{array}$ & $\begin{array}{l}\overrightarrow{0} \\
\text { D. } \\
\text { D. } \\
\end{array}$ & $\begin{array}{l}\stackrel{0}{\Xi} \\
\stackrel{\pi}{\pi} \\
2\end{array}$ & $\begin{array}{l}\overrightarrow{0} \\
\text { D } \\
\text { 这 }\end{array}$ & $\begin{array}{l}\overrightarrow{0} \\
0 \\
\stackrel{0}{0} \\
\text { D. }\end{array}$ & $\begin{array}{l}\frac{\varrho}{\pi} \\
\frac{\pi}{\pi} \\
2\end{array}$ \\
\hline $\begin{array}{c}\text { Group } 1 \\
\mathrm{n}(\%)\end{array}$ & $\begin{array}{c}27 \\
(30.7)\end{array}$ & $\begin{array}{c}14 \\
(40)\end{array}$ & $\begin{array}{l}\text { G1 vs G2 } \\
0.453\end{array}$ & $\begin{array}{c}36 \\
(31.9)\end{array}$ & $\begin{array}{c}5 \\
(50)\end{array}$ & $\begin{array}{l}\text { G1 vs G2 } \\
0.356\end{array}$ & $\begin{array}{c}41 \\
(33.6)\end{array}$ & $\begin{array}{c}0 \\
(0)\end{array}$ & $\begin{array}{l}\text { G1 vs } \\
\text { G2 }\end{array}$ \\
\hline $\begin{array}{c}\text { Group } 2 \\
\mathrm{n}(\%)\end{array}$ & $\begin{array}{c}31 \\
(35.2)\end{array}$ & $\begin{array}{c}10 \\
(28.5)\end{array}$ & $\begin{array}{c}\text { G1 vs G3 } \\
0.622\end{array}$ & $\begin{array}{c}39 \\
(34.5)\end{array}$ & $\begin{array}{c}2 \\
(20)\end{array}$ & $\begin{array}{l}\text { G1 vs G3 } \\
0.675\end{array}$ & $\begin{array}{c}40 \\
(2.8)\end{array}$ & $\begin{array}{c}1 \\
(100)\end{array}$ & $\begin{array}{l}1.000 \\
\mathrm{G} 2 \mathrm{vs}\end{array}$ \\
\hline $\begin{array}{c}\text { Group } 3 \\
\mathrm{n}(\%)\end{array}$ & $\begin{array}{c}30 \\
(34.1)\end{array}$ & $\begin{array}{c}11 \\
(31.5)\end{array}$ & $\begin{array}{l}\mathrm{G} 2 \text { vs G3 } \\
1.000\end{array}$ & $\begin{array}{c}38 \\
(33.6)\end{array}$ & $\begin{array}{c}3 \\
(30)\end{array}$ & $\begin{array}{c}\mathrm{G} 2 \text { vs G3 } \\
1.000\end{array}$ & $\begin{array}{c}41 \\
(33.6)\end{array}$ & $\begin{array}{c}0 \\
(0)\end{array}$ & $\begin{array}{c}\text { G3 } \\
0.494\end{array}$ \\
\hline $\begin{array}{l}\text { Total } \\
\mathrm{n}(\%)\end{array}$ & $\begin{array}{c}88 \\
(100)\end{array}$ & $\begin{array}{c}35 \\
(100)\end{array}$ & & $\begin{array}{c}113 \\
(100)\end{array}$ & $\begin{array}{c}10 \\
(100)\end{array}$ & & $\begin{array}{c}122 \\
(100)\end{array}$ & $\begin{array}{c}1 \\
(100)\end{array}$ & \\
\hline
\end{tabular}

The $\mathrm{p}$ values of the paired comparisons made using the Chi-square test in respect of complications. G1 = group 1 , $\mathrm{G} 2=$ group $2, \mathrm{G} 3=$ group 3

Table 5. p-values of VAS scores between groups calculated by Kruskal-Wallis test.

\begin{tabular}{ccccc}
\hline & VAS 1 & VAS 2 & VAS 3 & VAS 4 \\
\hline$p$ values & 0.152 & 0.178 & 0.323 & $\mathbf{0 . 0 3 9}$ \\
\hline
\end{tabular}

Table 6. $p$ values for pairwise comparisons of groups for each visual analog scale score

\begin{tabular}{lccc}
\hline & \multicolumn{3}{c}{$p$ values } \\
& Group 1 - Group 2 & Group 1 - Group 3 & Group 2 - Group 3 \\
\hline VAS 2 & 0.345 & 0.470 & 0.872 \\
VAS 3 & 0.508 & 0.114 & 0.346 \\
VAS 4 & $\mathbf{0 . 0 4 9}$ & 0.233 & $\mathbf{0 . 0 0 1}$ \\
\hline
\end{tabular}

be related to anal tonus and relatively greater anxiety before the procedure in younger patients. In studies by Peyromaure et al. [8] and Zisman et al. [9], a significant correlation was found between anxiety before the procedure and pain occurring during prostate biopsy.

When studies in literature that found no significant relationship between age and pain were examined, Hossack et al. [10], patients who had undergone biopsy with local anaesthesia were questioned about their preference for the same procedure or general anaesthesia/sedation for a potential second biopsy and it was reported that those who expressed a preference for general anaesthesia/sedation were younger patients. Zisman et al. [9] reported that even if there is no correlation between age and pain, those with pain persisting on the seventh day were significantly younger patients. In the current study, the latest pain score was measured at 1 hour after the procedure (VAS 4) and there was no significant relationship with age. This finding was attributed to the pain having been reduced to a great degree in the first hour with the effect of the anaesthesia applied (mean VAS 4 pain scores for Group 1, Group 2, Group 3 were 6, 1.9, 3.2, respectively). In a study by Inal et al. [11], although a negative correlation was reported between age and pain, it was not statistically significant. In that study, 6-12 cores sampling was applied depending on the 
prostate volume and the mean core number was 8.8 . Bastide et al. [12] also found no significant correlation between age and pain and the median core number was 7 (range: 4-10 cores). In the current study, a standard 12-core biopsy was applied to all patients. The difference between previous studies and the current study in the relationship between age and pain could be related to the number of cores taken.

In patients with prostate volume $>40 \mathrm{cc}$, Yun et al. [13] reported that pain scores during the procedure and at 20 mins after the procedure were significantly higher. The mean prostate volume of the 71 patients in that study was $42.2 \mathrm{cc}$, whereas in the current study the mean volume was $66.6 \mathrm{cc}$. However, no significant relationship was determined between prostate volume and any of the VAS scores in the current study when the groups were evaluated separately or together. Unlike the study of Yun et al. [13], our result was consistent with several studies in literature $[4,5,7,9$, $12,14]$.

From a scan of literature, no studies could be found that have reported a significant relationship between PSA levels and pain [4]. In the current study, a significant negative correlation was determined between both total and free PSA levels and the VAS 3 pain scores in Group 1. For entire cohort, although a similar negative correlation was seen for VAS 3, it was not statistically significant. A significant negative correlation was determined between the VAS 2 scores and free PSA for entire cohort (Table 3). To the best of our knowledge, this is the first study in literature to have shown a significant relationship between PSA levels and pain. However, as this relationship was not observed in all the groups there can be considered a need for further studies to investigate the relationship between PSA levels and pain.

In studies by Kaygisiz et al. [15], it was stated that however great the pain during digital rectal examination, then the pain occurring during probe placement and biopsy would be of the same degree. As far as we know, that is the only study that has evaluated the relationship between digital rectal examination and pain. In the current study, it was aimed to contribute to literature in a different aspect by evaluating the relationship between pain and digital rectal examination in respect of the examination findings. Accordingly, the VAS 4 pain scores of Group 2 patients with suspicious digital rectal examination findings were seen to be higher than those of the patients with benign findings (Table 3 ). When the results of the study by Kaygisiz et al. [15] are evaluated together with those of the current study, it can be concluded that it should be kept in mind that patients who experience greater pain during the digital rectal examination and have suspicious examination findings could feel more pain during the prostate biopsy.

The lower PSA value and the suspicious rectal examination findings as risk factors for pain may be considered as two opposite conditions. However, it is a clinically known fact that those may not always be in a relationship. We think that this result may be related to an underlying prostatitis in these patients, considering that suspicious digital rectal examination findings are not always associated with malignancy but may also be related to inflammation in the prostate. The increased risk of pain may also be due to this inflammatory condition in the prostate. However, we believe that this topic which is beyond the scope of this study should be evaluated with new studies.

In only one study that evaluated the relationship between pathology results and pain, no significant relationship was found [16]. In the current study, two contradictory results were seen related to the correlation between pain and the pathology results. In Group 3, the pain levels of those with benign pathology results were significantly higher than those of the patients with malignant pathology results in all the pain scores and the reverse of this was seen in Group 2 only in the VAS 2 score, suggesting that pain associated with the biopsy procedure was greater in those with benign pathologies compared to those with malignant pathologies (Table 2, Table 3). Nevertheless, despite this finding, when it is considered that the pathology results are unknown before the procedure, it is debatable whether the pathology results should be evaluated as a risk factor for pain occurring associated with the prostate biopsy. Djavan et al. [4] stated that patients with rectal bleeding experienced a significantly more uncomfortable procedure. Hossack et al. [10] reported that vasovagal syncope attacks were seen more in the group with higher pain scores compared to the other groups. However, no direct evaluation was made between pain and complications in either of these two 
studies. In the current study, when the groups were evaluated separately and together, no significant correlation was seen between pain and complications.

As much as we know, the relationship between education level and the pain occurring during prostate biopsy has not been previously evaluated. In the current study, education level was not seen to affect the pain scores when the groups were evaluated separately or together. However, values of borderline statistical significance were determined in Group 1 and Group 2 (Table 3). According to this, the pain scores during the procedure (VAS 2) of Group 1 patients with an education level of primary school and below, and the pain scores immediately after the procedure (VAS 3) of Group 2 patients with an education level of primary school and above, were seen to be higher. As these results showed borderline significance as a result of the evaluation of the relationship between pain and education level and because the results were contradictory, there can be considered to be a need for further studies on this subject.

There was no significant difference between the groups in terms of VAS 1, VAS 2 and VAS 3 pain scores (Table 5). VAS 4 pain scores were significantly lower in group 2 than in group 1 and group 3 (Table 6 ). We conclude that this result is due to the fact that levobupivacaine is longer effective than other agents.

\section{Limitations}

In our study, the small number of patients and the absence of all complications due to prostate biopsy were considered as limiting factors in our study.

\section{CONCLUSION}

The results of this study suggested that of all patients to be applied with prostate biopsy, those of a younger age, with a lower PSA level, with suspicious digital rectal examination findings and benign pathology results constitute a relatively higher risk group in respect of pain. The use of analgesia and/or anaesthesia methods which could be personalised beyond the routine protocols should be considered for patients with these risk factors. However, as the risk factors for pain occurring during prostate biopsy have been evaluated in a limited number of studies and as some of these risk factors have only been examined in this study, there is a need for further studies including a greater number of patients.

\section{Conflict of interest}

The authors disclosed no conflict of interest during the preparation or publication of this manuscript.

\section{Financing}

The authors disclosed that they did not receive any grant during conduction or writing of this study.

\section{REFERENCES}

1. Rietbergen JB, Kruger AEB, Kranse R, Schröder FH. Complications of transrectal ultrasound-guided systematic sextant biopsies of the prostate: evaluation of complication rates and risk factors within a population-based screening program. Urology 1997;49:875-80.

2. Collins GN, Lloyd SN, Hehir M, McKelvie GB. Multiple transrectal ultrasound-guided prostatic biopsies - true morbidity and patient acceptance. Br J Urol 1993;71:460-3.

3. Clements R, Aideyan OU, Griffiths GJ, Peeling WB. Side effects and patient acceptability of transrectal biopsy of the prostate. Clin Radiol 1993;47:125-6.

4. Djavan BOB, Waldert M, Zlotta A, Dobronski P, Seitz C, Remzi M, et al. Safety and morbidity of first and repeat transrectal ultrasound guided prostate needle biopsies: results of a prospective European prostate cancer detection study. J Urol 2001;166:856-60.

5. Chang SS, Alberts G, Wells N, Smith JR, Cookson MS. Intrarectal lidocaine during transrectal prostate biopsy: results of a prospective double-blind randomized trial. $\mathrm{J}$ Urol 2001;166:2178-80.

6. Philip J, Mccabe JE, Dutta Roy S, Samsudin A, Campbell IA, Javle P. Site of local anaesthesia in transrectal ultrasonography-guided 12-core prostate biopsy: does it make a difference? BJU Inter 2006;97:263-5.

7. Raaijmakers R, Kirkels WJ, Roobol MJ, Wildhagen MF, Schrder FH. Complication rates and risk factors of 5802 transrectal ultrasound-guided sextant biopsies of the prostate within a population-based screening program. Urology 2002; 60:826-30.

8. Peyromaure M, Ravery V, Messas A, Toublanc M, BocconGibod L, Boccon-Gibod L. Pain and morbidity of an extensive prostate 10-biopsy protocol: a prospective study in 289 patients. J Urol 2002; 167:218-21.

9. Zisman A, Leibovici DAN, Kleinmann J, Siegel YI, Lindner A. The impact of prostate biopsy on patient well-being: A prospective study of pain, anxiety and erectile dysfunction. J Urol 2001;165:445-54.

10. Hossack T, Woo HH. Acceptance of repeat transrectal ultrasonography guided prostate biopsies with local anaesthesia. 
BJU Int 2011;107:38-42.

11. Inal G, Yazici S, Adsan O, Ozturk B, Kosan M, Cetinkaya M. Effect of periprostatic nerve blockade before transrectal ultrasound-guided prostate biopsy on patient comfort: A randomized placebo controlled study. Int J Urol 2004;11:148-51. 12. Bastide C, Lechevallier E, Eghazarian C, Ortega JC, Coulange C. Tolerance of pain during transrectal ultrasoundguided biopsy of the prostate: risk factors. Prostate Cancer Prostatic Dis 2003;6:239-41.

13. Yun TJ, Lee J, Kim SH, Lee SE, Byun SS, Hong SK, et al. Prospective analysis on the relation between pain and prostate volume during transrectal prostate biopsy. Korean J Radiol 2007;8:231-5.
14. Rodriguez LV, Terris MK. Risks and complications of transrectal ultrasound guided prostate needle biopsy: A prospective study and review of the literature. J Urol 1998;60:2115-20.

15. Kaygisiz O, Inal G, Tas M, Ugurlu O, Oztur B, Adsan O. Can pain during digital rectal examination help us to decide the necessity and the method of anesthesia for transrectal ultrasound guided prostate needle biopsy?. Int Braz J Urol 2007; 33:470-6. 16. Saraçoğlu T, Unsal A, Taşkın F, Sevinçok L, Karaman CZ. The impact of pre-procedural waiting period and anxiety level on pain perception in patients undergoing transrectal ultrasoundguided prostate biopsy. Diagn Interv Radiol 2012;18:195-9. 